\title{
Trion-Induced Distinct Transient Behavior and Stokes Shift in $\mathrm{WS}_{2}$ Monolayers
}

Peng Fan ${ }^{a}$, Biyuan Zheng ${ }^{a}$, Xingxia Sun ${ }^{b}$, Weihao Zheng ${ }^{a}$, Zheyuan Xu ${ }^{a}$, Cuihuan $e^{a}$,

Yong Liu ${ }^{a}$, Xiujuan Zhuang ${ }^{a}$, Dong Li $^{b}$, Xiao Wang ${ }^{a}$, Xiaoli Zhu ${ }^{a}$, Ying Jiang ${ }^{* a}$, Anlian Pan $* b$

a. Key Laboratory for Micro-Nano Physics and Technology of Hunan Province,

School of Physics and Electronics, Hunan University, Changsha 410082, People’s

Republic of China.

b. Key Laboratory for Micro-Nano Physics and Technology of Hunan Province,

College of Materials Science and Engineering, Hunan University, Changsha 410082,

People's Republic of China.

\section{AUTHOR INFORMATION}

Corresponding Author:

Anlian Pan: anlian.pan@hnu.edu.cn

Ying Jiang: jying@,hnu.edu.cn 
1. AFM Image
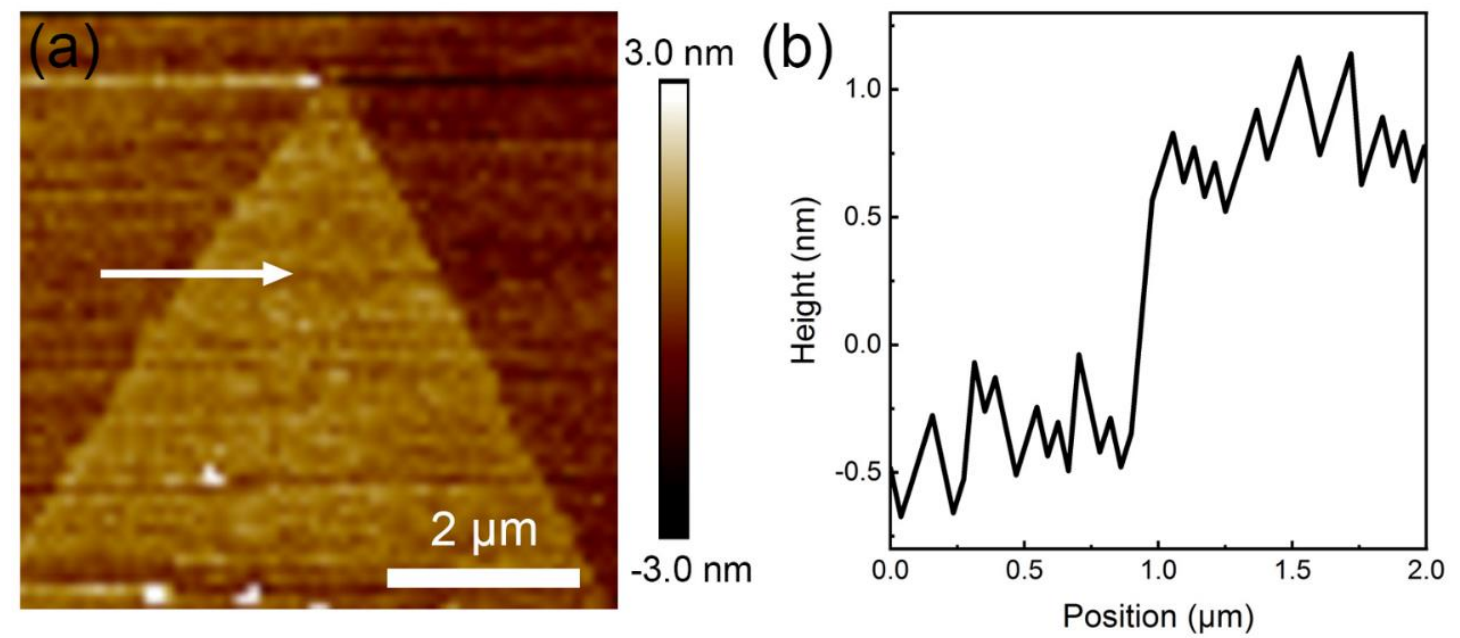

Fig. S1 The AFM image of $\mathrm{WS}_{2}$ monolayer as-grown on $\mathrm{Si} / \mathrm{SiO}_{2}$ substrate (a) and the line profile (b) as the line marked on (a).

\section{Steady-state Absorption Spectra}

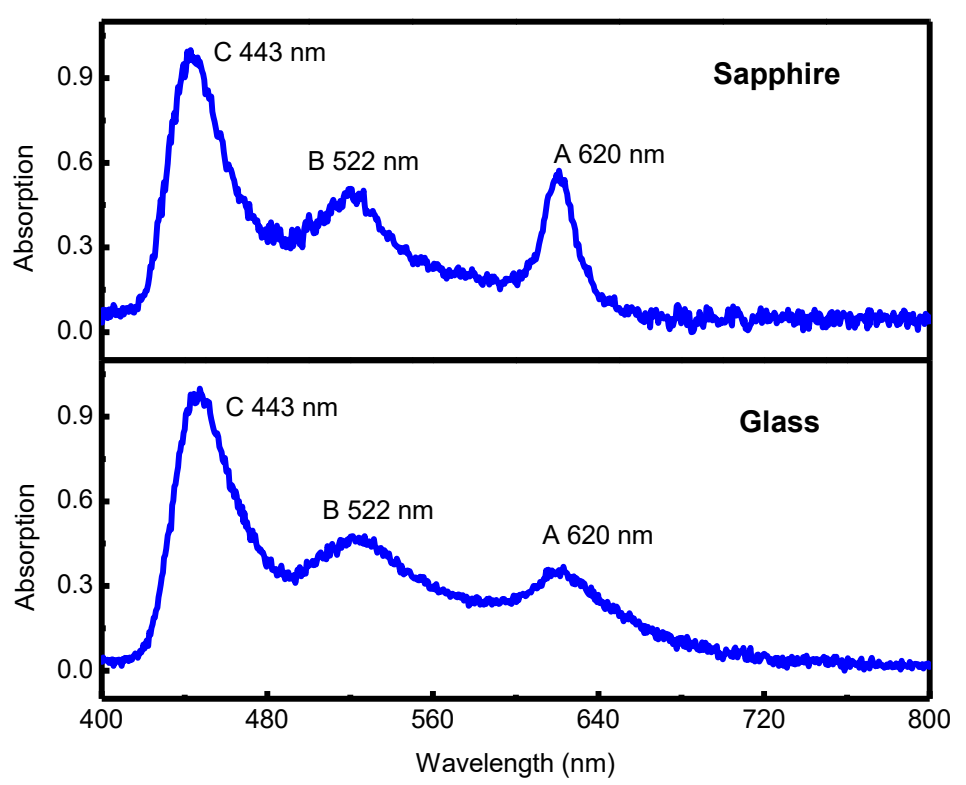

Fig. S2 The steady state absorption spectra of $\mathrm{WS}_{2}$ monolayer transferred onto (a) sapphire and (b) glass substrates, respectively. 


\section{Normalized PL Spectra}

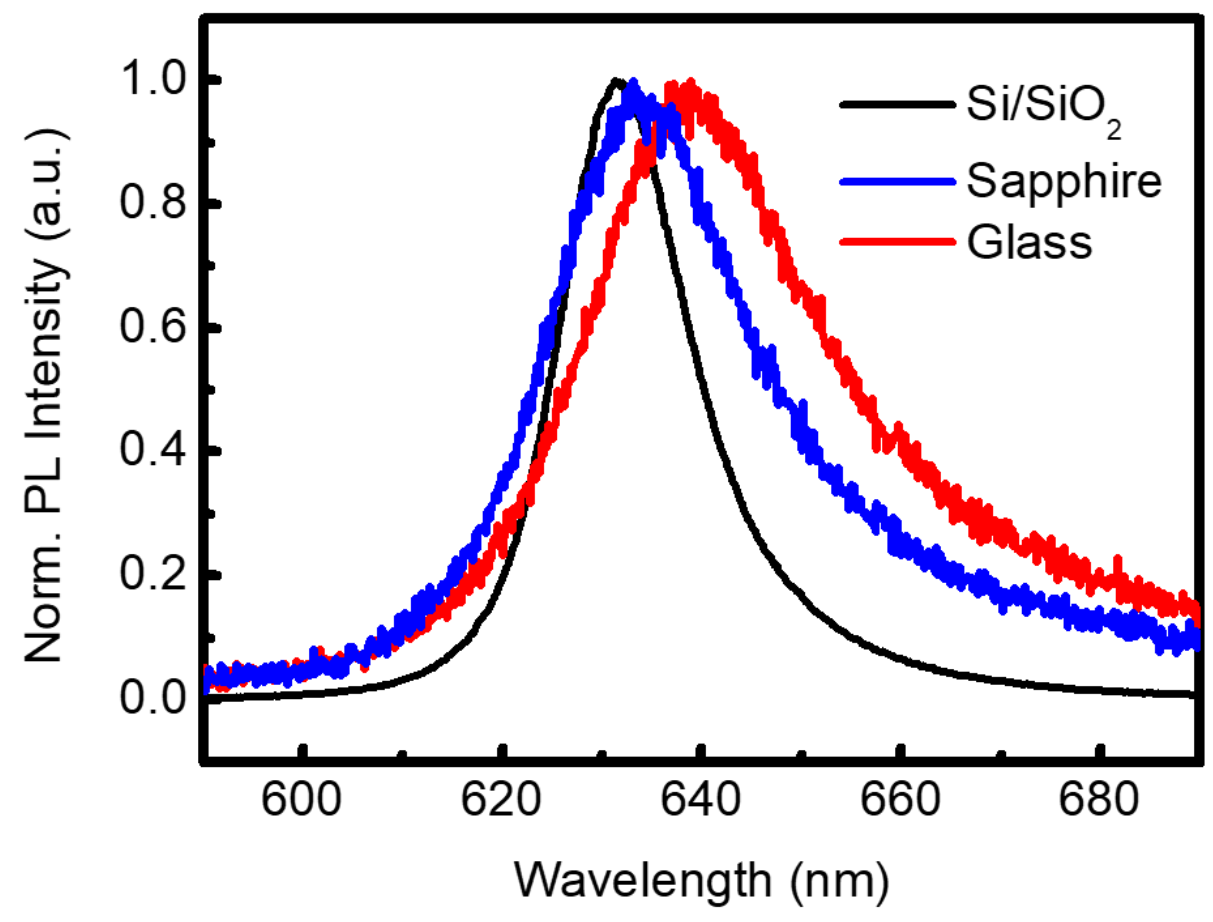

Fig. S3 The normalized PL spectra of $\mathrm{WS}_{2}$ monolayer as-grown on $\mathrm{Si} / \mathrm{SiO}_{2}$ substrate (black), transferred onto sapphire (red) and glass (blue) substrates, respectively. 
4. Steady-state Absorption Spectra (inverted for comparison) and DAS Components

(a)

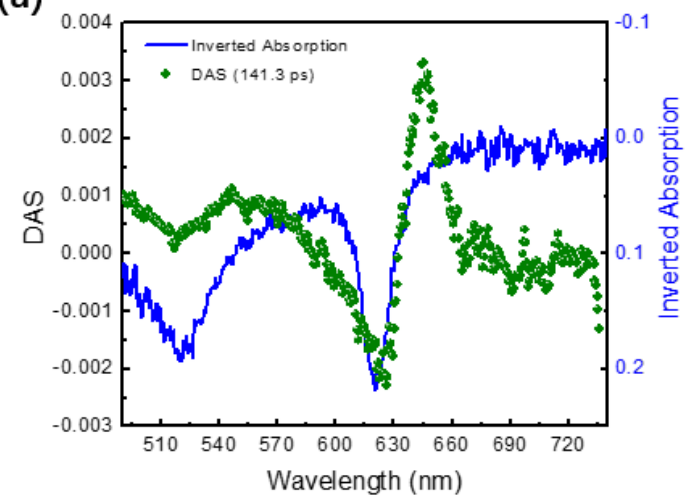

(b)

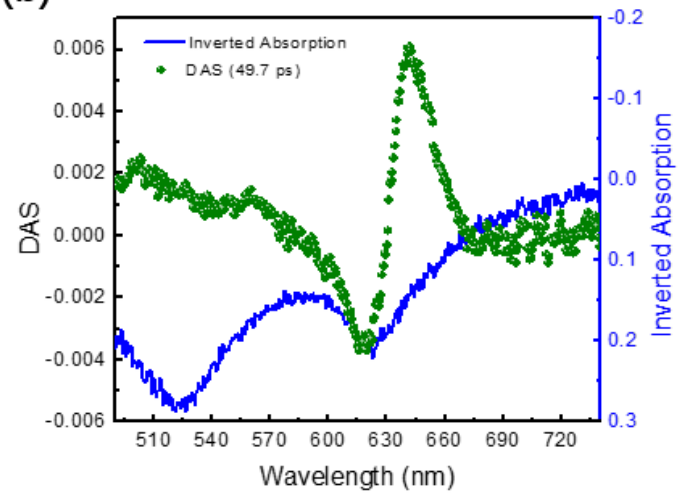

Fig. S4 The steady-state absorption spectra (inverted for comparison) and the DAS components responsible for exciton recombination in $\mathrm{WS}_{2}$ monolayers on sapphire (a) and glass (b) substrates, respectively.

\section{The Fast Two DAS Components from the Global Fitting}

(a)

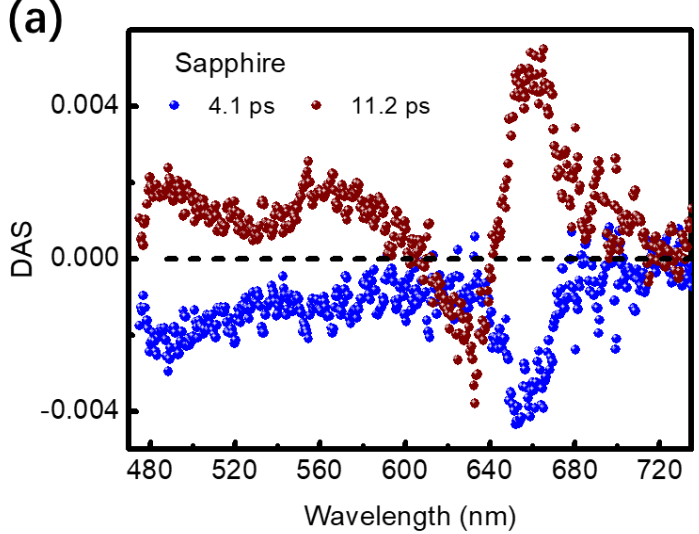

(b)

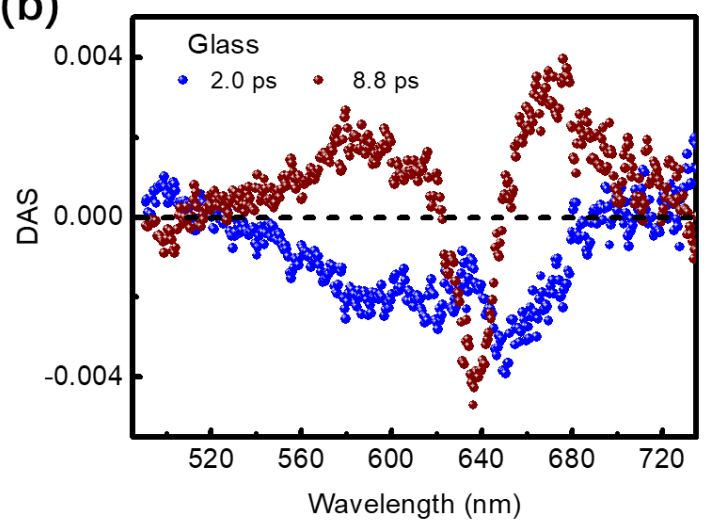

Fig. S5 The fast two DAS components in $\mathrm{WS}_{2}$ monolayers on sapphire (a) and glass

(b) substrates, respectively. 